\title{
Human brain activity time-locked to rapid eye movements during REM sleep
}

\author{
Satoru Miyauchi · Masaya Misaki · Shigeyuki Kan • \\ Takahide Fukunaga $\cdot$ Takahiko Koike
}

Received: 18 June 2008 / Accepted: 12 September 2008 / Published online: 2 October 2008

(C) The Author(s) 2008. This article is published with open access at Springerlink.com

\begin{abstract}
To identify the neural substrate of rapid eye movements (REMs) during REM sleep in humans, we conducted simultaneous functional magnetic resonance imaging (fMRI) and polysomnographic recording during REM sleep. Event-related fMRI analysis time-locked to the occurrence of REMs revealed that the pontine tegmentum, ventroposterior thalamus, primary visual cortex, putamen and limbic areas (the anterior cingulate, parahippocampal gyrus and amygdala) were activated in association with REMs. A control experiment during which subjects made self-paced saccades in total darkness showed no activation in the visual cortex. The REM-related activation of the primary visual cortex without visual input from the retina provides neural evidence for the existence of human pontogeniculo-occipital waves (PGO waves) and a link between REMs and dreaming. Furthermore, the time-course analysis of blood oxygenation level-dependent responses indicated that the activation of the pontine tegmentum, ventroposterior thalamus and primary visual cortex started before the
\end{abstract}

Electronic supplementary material The online version of this article (doi:10.1007/s00221-008-1579-2) contains supplementary material, which is available to authorized users.

S. Miyauchi $(\varangle) \cdot$ M. Misaki $\cdot$ S. Kan · T. Fukunaga $\cdot$ T. Koike Kobe Advanced ICT Research Center,

National Institute of Information and Communications

Technology (NICT), 588-2 Iwaoka, Iwaoka-cho,

Nishi-ku, Kobe, Hyogo 651-2492, Japan

e-mail: miyauchi@po.nict.go.jp

S. Miyauchi $\cdot$ S. Kan $\cdot$ T. Koike

CREST, Japan Science and Technology Agency (JST),

Kawaguchi, Japan

M. Misaki

Japan Society for the Promotion of Science (JSPS), Tokyo, Japan occurrence of REMs. On the other hand, the activation of the putamen and limbic areas accompanied REMs. The activation of the parahippocampal gyrus and amygdala simultaneously with REMs suggests that REMs and/or their generating mechanism are not merely an epiphenomenon of PGO waves, but may be linked to the triggering activation of these areas.

Keywords REM sleep · Rapid eye movements · Saccade · Functional magnetic resonance imaging $\cdot$ Dreaming

\section{Introduction}

It is well known that our eyes move frequently during certain periods of sleep (rapid eye movements: REMs) and there is a high incidence of vivid dreams during REM sleep. There is a long-standing debate about whether REMs are functionally equivalent to waking saccades and about whether there is a relationship between the visual content of a dream and REMs in humans (Dement and Kleitman 1957; Roffwarg et al. 1962; Hobson and McCarley 1977; Doricchi et al. 2007). A number of imaging and psychophysiological studies have addressed this issue using a variety of investigative approaches in humans. Electroencephalography (EEG) and magnetoencephalography (MEG) studies have found REM-related neural activity in the visual cortex where no such activity was seen after waking saccades in total darkness (Miyauchi et al. 1990; Ioannides et al. 2004; Ogawa et al. 2005). However, the exact location of this activity is still unclear, partly because the inverse problem of estimating the current sources on the basis of EEG/MEG data alone is fundamentally ill-posed, and partly because the deep structural resolution of EEG/MEG is variable (Baillet et al. 2001). 
Several positron emission tomography (PET) studies have revealed activations in the visual cortex during REM sleep (Maquet et al. 1996; Braun et al. 1998) and a positive correlation between REM density and the activation of the visual cortex (Braun et al. 1998; Peigneux et al. 2001). In addition, a recent fMRI study succeeded in separating two distinct functional sub-states (tonic and phasic periods) within REM sleep in humans (Wehrle et al. 2007). Nevertheless, the lack of temporal resolution makes PET scans and non-event-related fMRI analysis unable to distinguish between tonic activations throughout REM sleep and phasic activations temporally related to REMs. Taking a different approach to this question, Doricchi et al. carried out neuropsychological studies in which the functional equivalence between REMs and waking saccades was investigated in spatial hemineglect patients (Doricchi et al. 1993, 1996, 2007). On the basis of their findings, they showed that morphologically different types of REMs exist in human REM sleep. Although their results and "evolutionary hypothesis" (Doricchi et al. 2007) are intriguing, they are not yet conclusive. After all, the relationship between dreaming and REMs remains an open question (Hobson et al. 2000). Thus, notwithstanding several lines of evidence provided by neurophysiological and neuropsychological studies, the relationship between dreaming and REMs deserves further study. In particular, an event-related fMRI approach may offer more precise findings and hypotheses than previous studies.

Another important question concerning REM sleep is whether ponto-geniculo-occipital (PGO) waves occur in humans. PGO waves, named for the brain structures where these waves appear most prominently (the pons, lateral geniculate nucleus, and occipital cortex), are one of the phasic events of REM sleep that include REM bursts and have been observed in a variety of mammals. It is thought that PGO waves are related to several important brain functions such as sensorimotor integration, dreaming, learning, development of the visual system and so on (Callaway et al. 1987; Marks et al. 1995; Datta 2000). Although the existence of PGO waves in humans has been suggested by several studies based on direct depth recordings (Salzarulo et al. 1975; Lim et al. 2007), MEG (Ioannides et al. 2004), PET (Peigneux et al. 2001), and fMRI (Wehrle et al. 2005), there is a need for higher spatial and temporal resolution measurements to elucidate the cortical-subcortical functional neuroanatomy of PGO waves.

To address these issues, we recorded polysomnogram and fMRI data in normal subjects while they slept in an MR scanner. We then examined the cortical-subcortical network activity that coincided with REMs and the temporal characteristics of the blood oxygenation level-dependent (BOLD) responses.

\section{Materials and methods}

\section{Participants}

Seventeen normal volunteers (14 males and 3 females, mean age 25 years, range 22-31 years) participated in this study. All subjects had normal or corrected-to-normal visual acuity, normal eye movements, and were free of psychiatric/sleep disorders and medication. All subjects gave informed written consent to participate. The present study was approved by the ethics committee of the National Institute of Information and Communications Technology (Japan) and was in accordance with the Declaration of Helsinki.

\section{Procedure}

All sleep experiments started between 3:00 AM and 6:00 $\mathrm{AM}$, the period when REM sleep tends to occur most frequently. Subjects were required to stay awake all night until the start of the experiment, and they slept inside the MR scanner for two consecutive nights. They were instructed to refrain from alcohol and caffeine-containing beverages for $24 \mathrm{~h}$ prior to the experiment. The subjects wore earplugs and put on soundproof headphones to muffle the scan noise. They lay on an elastic mattress on the scanner's table. In addition, the subjects were habituated to the scan noise by being exposed to a recording of the scan noise for at least $6 \mathrm{~h}$ before sleep. The magnet room was kept completely dark and no stimulus was presented. Subjects who showed distinct REM sleep in the MR scanner also participated in a control experiment during which subjects made self-paced saccades in total darkness.

Polysomnographic recording and data analysis

Twenty-four channels of EEG, vertical and horizontal electroocculography (EOG), submental electromyogrpahy (EMG), and electrocardiography (ECG) were acquired using an MRI-compatible amplifier (BrainAmp MR, Brain Products $\mathrm{GmbH}$, Munich, Germany) and an electrode cap (Falk Minow Services, Herrsching-Breitbrunn, Germany) with $\mathrm{Ag} / \mathrm{AgCl}$ ring electrodes. A reference electrode was placed at the middle point between $\mathrm{Fz}$ and $\mathrm{Cz}$. A raw record was sampled at $5 \mathrm{kHz}$ (bandpass filtered between 0.016 and $250 \mathrm{~Hz}$ ) using Brain Vision Recorder software (Brain Products $\mathrm{GmbH}$ ). Brain Vision Analyzer software (Brain Products $\mathrm{GmbH}$ ) was used for offline correction of scanning artifacts and ballistocardiogram (BCG) artifacts (see Supplementary materials). Horizontal EOG was recorded from both eyes with $\mathrm{Ag} / \mathrm{AgCl}$ ring electrodes placed at the external canthi, and vertical EOG was recorded from the right eye with $\mathrm{Ag} / \mathrm{AgCl}$ ring electrodes placed at the suborbital 
and Fp2. After down-sampling to $200 \mathrm{~Hz}$, the polysomnographic data were exported to an in-house MATLAB (Mathworks, Inc., Sherborn, MA, USA) program for further analysis. The reference channel was digitally replaced with an average of TP9 and TP10 electrodes, located behind the ears. The polysomnographic recording was scored visually by an experienced sleep scorer (SM) according to standard criteria (Rechtschaffen and Kales 1968). In addition to the polysomnographic recording, eye movements and body movements were monitored using an MRI-compatible remote infrared eye tracking system and infrared cameras (see Supplementary materials).

\section{fMRI data acquisition and analysis}

The fMRI measurements were performed using a 1.5-T scanner (MAGNETOM Vision plus, Siemens, Germany) simultaneously with the polysomnographic recording. T2*weighted images (a gradient echo single-shot EPI sequence) were acquired from 24 slices (parallel to the ACPC line; $4 \times 4 \times 5$-mm voxels with no gap; TE $=66 \mathrm{~ms}$; $\mathrm{FA}=90^{\circ} ; \mathrm{TR}=4 \mathrm{~s}$, acquisition time $=2.6 \mathrm{~s}$ ) covering the whole brain. In the sleep experiment, each recording session consisted of 825 scans $(55 \mathrm{~min})$. We repeated the sessions successively and stopped when the subject was completely awake or complained of discomfort in the scanner. The subjects were queried after the end of the last recording session about whether they had dreams, however, we did not wake the subject from each REM sleep to obtain a dream report, because stable REM sleep in the MR scanner does not occur frequently and it was difficult for the subject to fall asleep again once he/she was woken up. Head movement was minimized through mild restraint and cushioning. For all subjects, a T1-weighted 3D MP RAGE scan $(1 \times 1 \times 1 \mathrm{~mm}$ voxels $)$ covering the entire brain was also recorded to identify brain sulci in each subject. fMRI data were preprocessed and analyzed using SPM2 (Wellcome Department of Cognitive Neurology, http:// www.fil.ion.ucl.ac.uk/spm/). fMRI data that did not contain REM sleep and data before and after REM sleep were discarded. After correcting for differences in slice timing within each image volume, each scan was aligned with the first remaining scan, normalized according to the Montreal Neurological Institute (MNI) template, and spatially smoothed with an $8 \times 8 \times 10 \mathrm{~mm}$ FWHM Gaussian kernel. In addition, data from subjects showing head movements greater than $3 \mathrm{~mm}$ were excluded from further analysis. The signal time-course was high-pass filtered (cutoff frequency: $0.0078 \mathrm{~Hz}$ ) to remove low-frequency drifts.

After the preprocessing, event-related analysis was done using the standard hemodynamic response function (canonical HRF) and its temporal derivative and dispersion (provided in SPM2) with the six realign parameters for motion correction. The significance criterion was $P<0.001$ (uncorrected). The event onset was defined as the time at the onset of each REM. REM was defined as EOG change with an amplitude of more than $50 \mu \mathrm{V}$ and duration of less than $300 \mathrm{~ms}$. In addition, each REM was verified using the video recording of eye (eyelid) movements, and discarded if the corresponding eye movement could not be observed.

To see whether saccades in total darkness activate visual cortices, we conducted another fMRI experiment. Ten of the 13 subjects participated in this experiment. In the saccades experiment, no visual stimulus was presented in total darkness. The subjects were required to keep their eyes closed throughout the experiment. Before the experiment, saccade frequency and size were practiced by the instruction of an experimenter observing subjects' eye movements using EOG and images of eyelid movements on the monitor, though it was difficult to control saccade size in total darkness. We employed a block design for the saccade experiment because it is more effective for detecting activations in short-period experiments. The test block during which they were instructed to make large horizontal saccades every $1-3 \mathrm{~s}$ at a self-pace (20 s) was alternated with the control block (20 s) in which they were asked not to move their eyes. Each block was preceded by a tactile stimulus to the index finger provided by an air-puff stimulator that informed the subjects to either move their eyes or keep their eyes still. A pair of task and control blocks was repeated six times in a run. Each subject performed three runs. All the measurements were conducted after adaptation to total darkness for $15 \mathrm{~min}$ to eliminate the effect of afterimages. Eye movements were monitored by the infrared camera of the eye tracking system and EOG recording. The mean number of saccades per block was 18.0 (SD 3.2). The first five image volumes were discarded from each run to allow for T1 equilibration effects. Activated voxels in each experimental condition were identified using a statistical model containing boxcar waveforms convolved with a canonical hemodynamic response function. Other procedures were the same as those of the sleep experiment.

For group analysis, a random-effects model was applied, and the significance threshold was set at $P<0.05$ [familywise error (FWE) corrected]. To obtain the Talairach coordinates of the activation, we searched for local maxima that were at least $4 \mathrm{~mm}$ apart in the significant cluster, and their MNI coordinates were converted using a nonlinear transformation method (http://www.mrc-cbu.cam.ac.uk/Imaging/Common/mnispace.shtml).

Time course analysis and estimation of the onset time of the BOLD response

To determine the temporal characteristics of the BOLD response to REM onset in each activated area, we com- 
Fig. 1 fMRI activity that was temporally related to REMs. Numbers $1-7$ in slices indicate activated areas: 1 pontine tegmentum, 2 ventroposterior thalamus, 3 primary visual cortex, 4 putamen, 5 anterior cingulate cortex, 6 parahippocampal gyrus and 7 amygdala. $L$ left, $R$ right
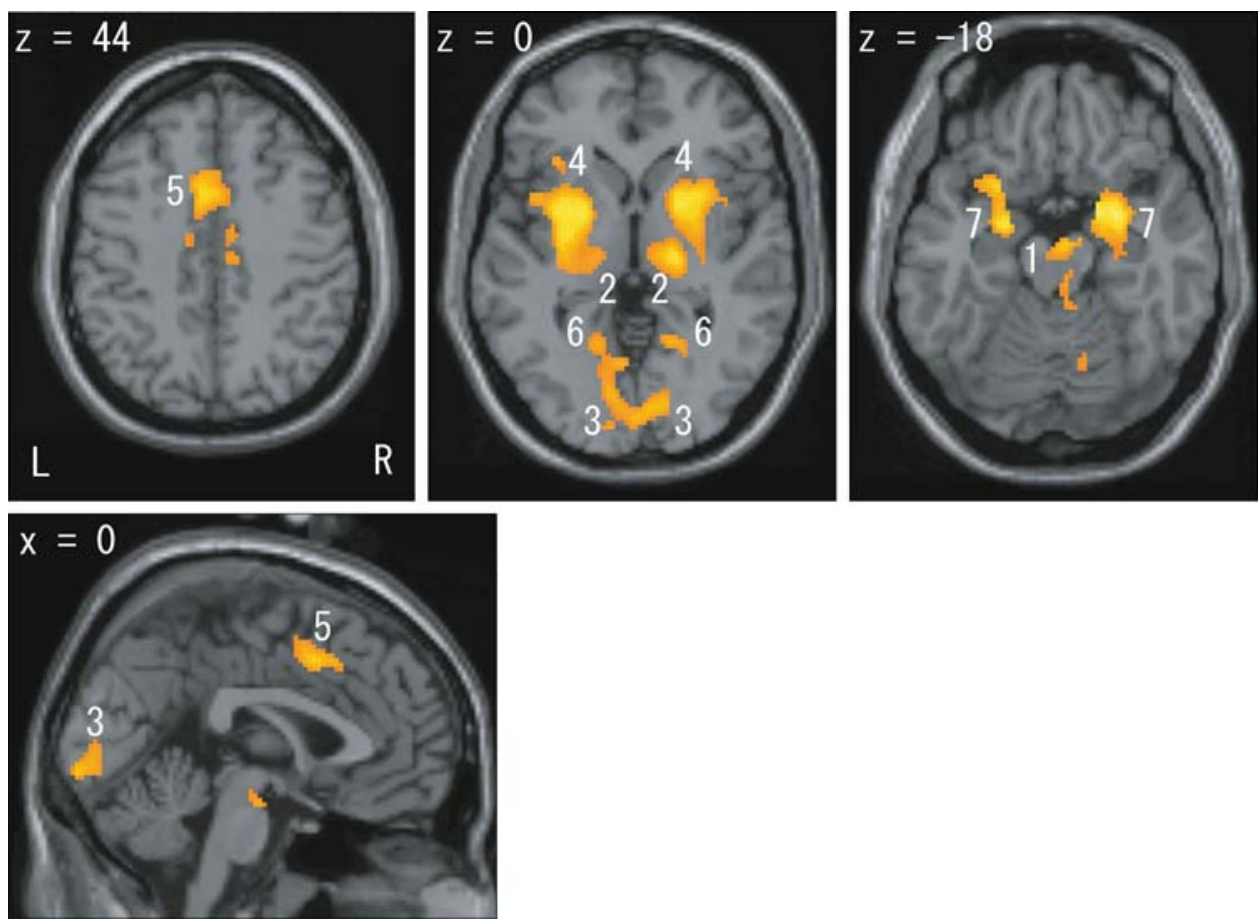

puted the average time courses of the BOLD signals timelocked to REM onset. The preprocessed fMRI data, ranging from $8 \mathrm{~s}$ before to $32 \mathrm{~s}$ after REM onset, were grand-averaged across all subjects (see Supplementary materials for details).

Furthermore, to confirm that the BOLD response timelocked to REM onset was not due to incidental fluctuation, we created 'pseudo REMs' during the REM sleep period, and computed grand-averaged time courses of the BOLD signals (13 pseudo REMs/subject, 169 pseudo REMs in total). Based on quasi-random numbers, pseudo REMs were interspersed within the period during which REMs appeared infrequently. If a pseudo REM happened to be in the 4-s epoch (correponding to fMRI acquisition time) during which actual REMs appeared, in the epoch before, or in one of the two epochs following actual REMs, it was skipped and a newly generated pseudo REM was set.

\section{Results}

Subjects slept inside the scanner for $2-7 \mathrm{~h}$ each night. Thirteen (11 males and 2 females) of the subjects reached stable REM sleep at least once, and this lasted 19-53 min on the second night. Although all subjects showed slowwave sleep, and some of them showed fragmented REM sleep during the first night, no subject reached stable REM sleep and woke up after $2-3$ recording sessions. As a result, most REM sleep was deprived on the first night, which might have facilitated the appearance of REM sleep on the second night. Thus, we conducted an event-related fMRI analysis time-locked to REMs that occurred during REM sleep on the second night. Particular care was taken to identify pure rapid eye movements and their onset time through simultaneous visual inspection of EOGs and video recordings of eye (eyelid) movements. In total, 1,082 REMs from the 13 subjects were regarded as events.

\section{Activation associated with REMs}

As shown in Fig. 1, the random effects group analysis based on 13 subjects revealed significant activation $(P<0.05$, FWE corrected) accompanying REMs in the pontine tegmentum [Talairach coordinates $(x, y, z$ and $Z$-score) of the peak foci of activation: $6,-11,-16 ; 5.55]$, the bilateral ventroposterior thalamus (right: $20,-17,3 ; 5.84$; left: -16 , $-17,35.15$ ), probably including the lateral geniculate nucleus, and the bilateral primary visual cortex (V1) (right: $8,-83,6 ; 5.4$; left: $-2,91,-0 ; 5.57)$. In addition, bilateral activations were found in the parahippocampal gyrus (PHG) (right: $12,-10,-15 ; 5.19$, Brodmann's area (BA) 34; left: $-22,-24,-9 ; 5.6$, BA 35), putamen (right: 24,4 , 5; 6.36; left: $-28,4,-7 ; 6.16)$ extending to the insula, medial frontal areas from anterior cingulate cortex (ACC) (right: $10,-4,46 ; 5.13$, BA 24; left: $-4,14,40 ; 5.61$, BA 32 ), and amygdala (right: $30,-1,-12 ; 6.49$; left: $-22,-5$, $-13 ; 6.04)$. There was no brain area that showed a negative BOLD response. 


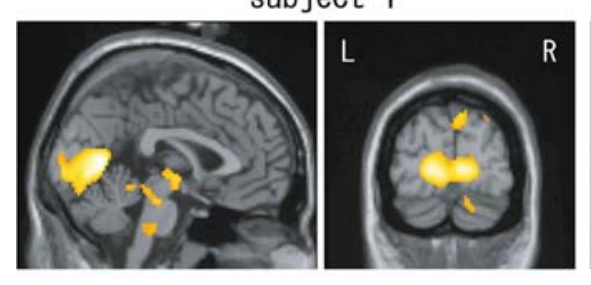

subject 4
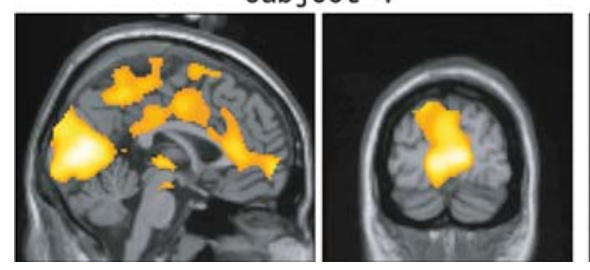

subject 2

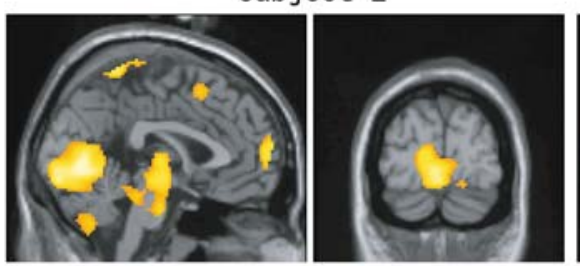

subject 5
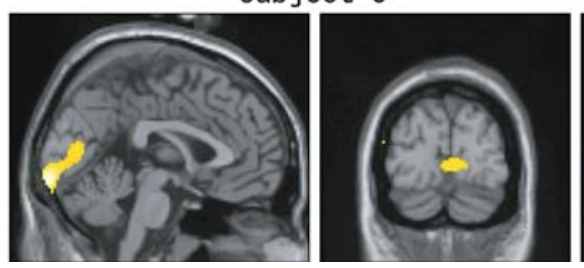

subject 3

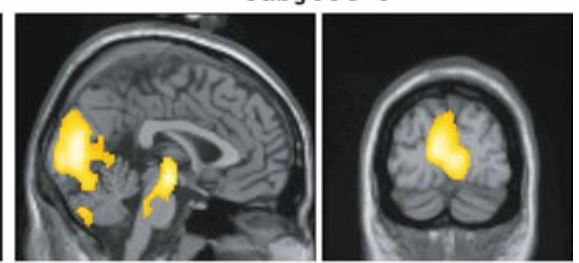

subject 6

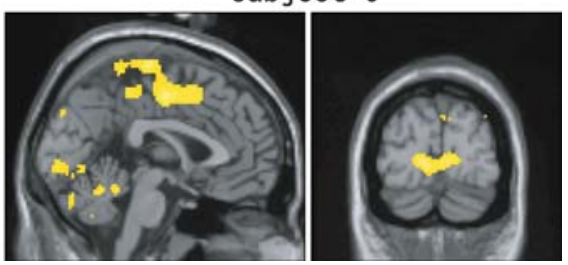

Fig. 2 Individual activation maps displayed as sagittal $(x=0)$ and coronal $(y=-88)$ sections of six representative subjects $(P<0.001$, uncorrected)

Figure 2 is the result of the individual analysis of six representative subjects $(P<0.001$, uncorrected) and shows the individual differences in the activation of the visual cortex. All subjects showed activation of V1. However, whereas the activation was confined in the calcarine fissure in some subjects (e.g., sub. 5 and 6), other subjects showed activation extending to the extrastriate cortex (e.g., sub. 3 and 4).

Activation associated with saccades in total darkness

Saccades in total darkness activated several brain areas. As shown in Fig. 3, in addition to the activations of the bilateral frontal eye field (right: 44, $-9,45 ; 15.57$, BA 6; left: $-36,-10,43,16.74$, BA 6), the left ACC and supplementary eye field (SEF) $(2,3,53,15.64)$ extending to the right corresponding areas and the bilateral putamen (right: 26, $-10,0,11.45$; left: $-24,-6,-1,10.55)$ extending to the insula were activated, as is the case with REMs. However, no activation was found in the visual cortices, PHG or amygdala $(P<0.05$, FWE corrected $)$, even when the threshold was lowered $(t=3.09$, corresponding to $P<0.001$, uncorrected).
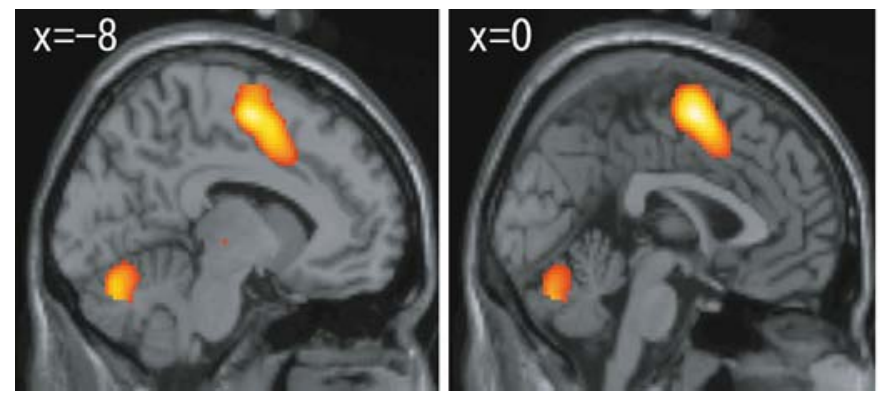

Fig. 3 Activation associated with voluntary saccades in total darkness. In addition to the bilateral frontal eye field (not shown here), the left anterior cingulate cortex and SEF extending to the right corre-
Time course analysis of BOLD response accompanying REMs

To determine the temporal characteristics of the BOLD response to REM onset in each activated area, we computed the grand-averaged time courses of the BOLD signals timelocked to REM onset and estimated the onset time of the BOLD response (see "Materials and methods"). Since the BOLD response is much slower than the corresponding neural activity, we used only the first REM of REM bursts and isolated REMs, before which there were no discernible eye movements for at least $8 \mathrm{~s}$. This was done to avoid the confounding effect of a BOLD response generated by a preceding REM. In total, BOLD time courses of 168 REMs were averaged. On the basis of the group analysis results, a set of regions of interest (ROIs), which consisted of all the voxels exceeding a statistical threshold $(P<0.001$, uncorrected) in the individual analysis, was defined for each subject; the ROIs included the pontine tegmentum, the bilateral ventroposterior thalamus, the bilateral posterior half of the calcarine fissure, the bilateral PHG, the bilateral ACC, the bilateral putamen, and the bilateral amygdala. The
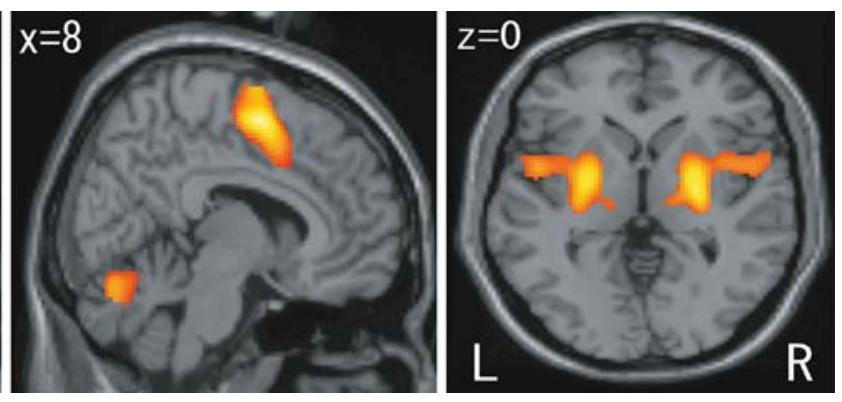

sponding areas and the bilateral putamen extending to the insula were activated. However, no activation was found in the visual cortices 
grand-averaged time courses of the BOLD response timelocked to REM onset revealed that the BOLD onset in the pontine tegmentum, ventroposterior thalamus and V1 preceded REM onset by at least a few seconds. For quantification, we fitted a response function to the sampled data and estimated the onset time of the BOLD response in each area (Fig. 4a). Whereas the response onset time in these areas preceded REM onset (pontine tegmentum: $-4.7 \mathrm{~s}$ from REM onset, ventroposterior thalamus: $-3.8 \mathrm{~s}, \mathrm{~V} 1:-2.8 \mathrm{~s})$, activation in PHG $(0.8 \mathrm{~s})$, ACC $(0.6 \mathrm{~s})$, putamen $(-0.6 \mathrm{~s})$, and amygdala (1.4 s) accompanied REM onset (Fig. 4b).

As for the time course time-locked to pseudo REMs, no discernible signal changes were observed in the areas that were activated in a time-locked manner to actual REMs (Fig. 4a, blue solid line).

\section{Discussion}

Using simultaneous fMRI and polysomnographic recording during REM sleep and event-related analysis time-locked to the occurrence of REMs, we found that activation of the pontine tegmentum, ventroposterior thalamus, and V1 started before REM onset, whereas activation of the putamen, ACC, PHG and amygdala accompanied REMs. In addition, although self-paced saccades in total darkness also activated the putamen and ACC, no activation was found in the visual cortex or other limbic and subcortical areas.

\section{Activation preceding REM}

The pontine tegmentum, ventroposterior thalamus, and V1 (the PGO areas) are brain areas where PGO waves appear most prominently. It is well known that REM occurrence is highly correlated with PGO waves in the cat, though the temporal relation of PGO waves to REMs in humans is not yet clear. Therefore, these activations most likely originate in PGO waves during REM sleep. These results are quite consistent with those of a recent fMRI study, which reported activations of the ventroposterior thalamus and V2 accompanying REMs (Wehrle et al. 2005). Why we found activation in V1 and the pontine tegmentum, but not in V2,
Fig. 4 Time course analysis of BOLD response accompanying REMs and pseudo REMs. a Grand-averaged time courses (solid black traces) with SE and estimated BOLD response (solid red traces) in each ROI, which consisted of all the voxels exceeding a statistical threshold in the individual analysis $(P<0.001$, uncorrected). The estimated BOLD onset from REM onset and its 95\% confidence interval are indicated by the red dashed line and the gray band, respectively, in each graph. The blue solid line indicates grand-averaged time courses of BOLD response timelocked to pseudo REMs. b The estimated BOLD onset from REM onset derived from (a) and its SE. The asterisks indicate that the estimated BOLD onset was significantly earlier than REM onset (onset time $=0$, $P<0.05)$
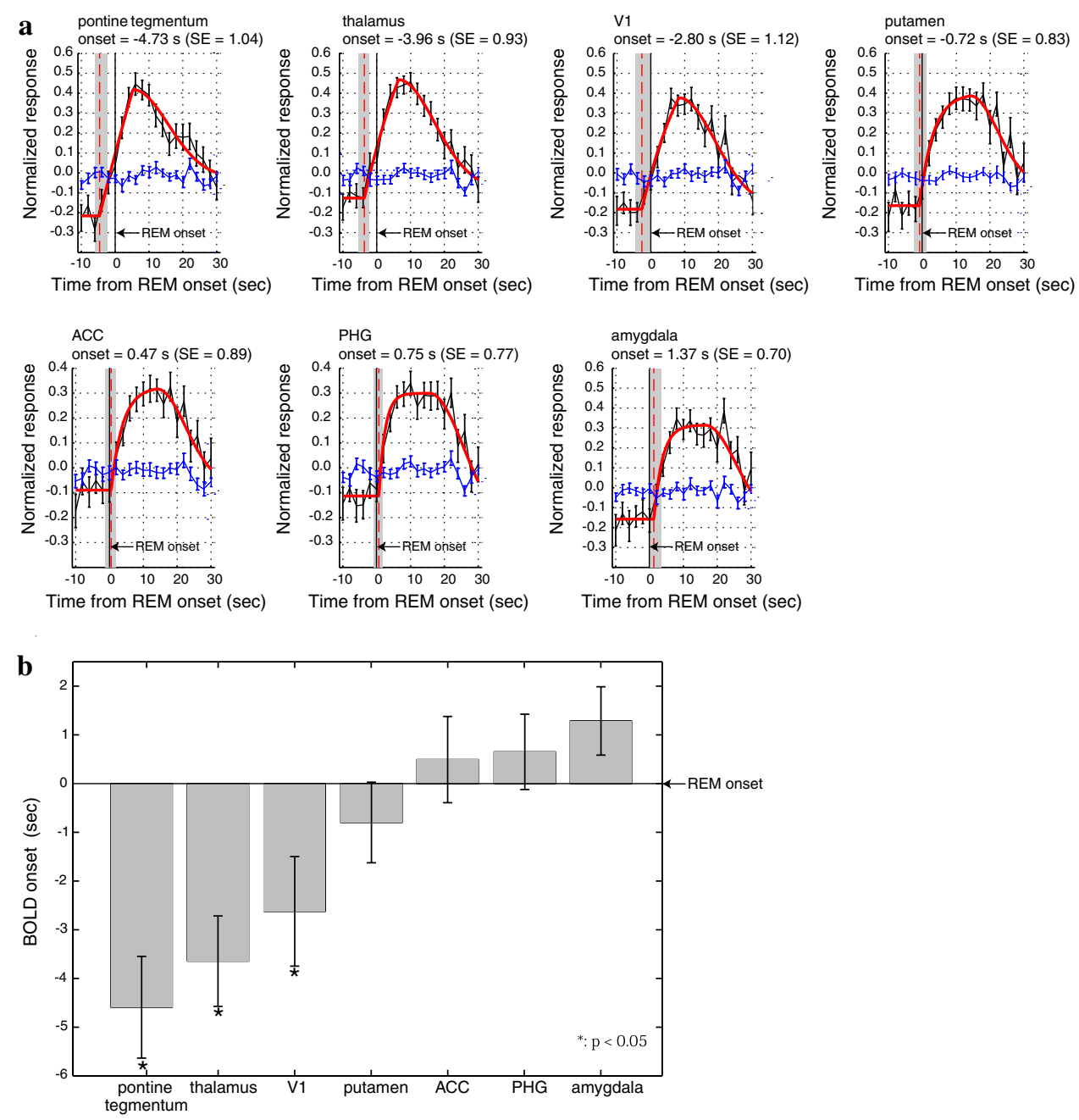
is not clear. The difference may be due to differing analysis methods. Whereas we used event-related analysis timelocked to the occurrence of more than 1,000 REMs, they used the absolute number of REMs per 3-s epoch as a regressor and analyzed relatively few fMRI scans obtained from three subjects.

No activation was found in the visual cortex accompanying self-paced saccades in our study. Several fMRI/PET studies, however, have reported activation of the visual cortex accompanying saccades in total darkness, suggesting that efferent copy signals of eye movements activate the visual cortex (Bodis-Wollner et al. 1997; Sylvester and Rees 2006). Although the reason for this inconsistency is not clear, Redcay et al. (2007) suggested the possibility that the responsiveness of the visual cortex to visual stimuli depends on whether the subject's eyes are open or closed. Namely, the visual cortex of subjects with eyes closed tended to show a BOLD signal decrease rather than increase in response to visual stimuli. In most studies that reported activation of the visual cortex accompanying saccades in total darkness, subjects were required to keep their eyes open. On the contrary, our subjects were required to keep their eyes closed in order to avoid misinformed activation by blink (Kato and Miyauchi 2003; Bristow et al. 2005) and to equalize the physical condition of the eyes between the saccade experiment and REM sleep experiment. Therefore, saccades with closed eyes might underlie the lack of activation in the visual cortex in the present experiment. In any case, even at the lower threshold, there was no activation in the visual cortex accompanying selfpaced saccades in total darkness in the present block-design experiment during which subjects closed their eyes, as during the REM sleep experiment. Furthermore, compared to event-related designs which we employed in the REM sleep experiment, block designs have superior statistical power (Friston et al. 1999) and higher detectability of activations (Birn et al. 2002). Considered together, the result of the self-paced saccade experiment indicates that the activation of V1 time-locked to the REMs was not caused by efferent copy signals accompanying the REMs, but rather a functional link between REMs and visual activities during REM sleep, that is, dreaming.

V1 was activated preceding REMs, though there was no visual input from the retina. Several studies report that visual imagery shares many of the functional properties and cortical structures, especially V1, used in visual perception (Kosslyn et al. 1999; Klein et al. 2000). When taken together with findings of a high correlation between the number of REMs and visual imagery in dreaming (Hong et al. 1997), and that eye movements during visual imagery are not random but reflect the content of the visualized scene (Brandt and Stark 1997; Laeng and Teodorescu 2002), the activation of $\mathrm{V} 1$ before REM onset suggests that dream imagery, probably driven by PGO waves, occurs before REM and that REM may occur in order to scan the dream imagery. Furthermore, as shown in Fig. 2, there was considerable individual difference in the extent of the activation in the visual cortex. Since the number of events (REMs) and the number of analyzed scans were different in each subject, strict comparison of the activation among individuals was difficult. Even so, whereas some subjects showed activation confined to the calcarine fissure, other subjects showed more extending activation to the extrastriate cortex. These individual differences may reflect differences in dream content and vividness. On the other hand, the result of the group analysis showing the $\mathrm{V} 1$ activation indicates that $\mathrm{V} 1$ is the common activated area across subjects.

Several questions remain, however. Unlike psychological studies on dreaming in natural sleep, we did not wake the subject from each REM period and obtain a dream report, because stable REM sleep in the MR scanner does not appear frequently. In addition, the activation of the visual cortex in the present study was obtained from the analysis of a great volume of fMRI data associated with more than 1,000 REMs. Therefore, we cannot directly affirm the relationship between the direction of REMs and the gaze of the dreamer at the dream scene, as proposed in the "scanning hypothesis" (Dement and Kleitman 1957). In relation to the scanning hypothesis, however, Doricchi et al. (2007) recently reported that a left unilateral neglect patient showed the selective disappearance of the optokinetic nystagmus $(\mathrm{OKN})$ and Nystagmoid-REMs in one horizontal direction, suggesting that morphologically different types of REMs exist in humans: Nystagmoid-REMs, which share phylogenetically ancient oculomotor mechanisms with OKN, and otherwise. V1 showed distinct activation in both the event-related analysis and the time-course analysis. In the time-course analysis, we selected the first REM of REM bursts and isolated REMs to avoid the confounding effect of a BOLD response generated by a preceding REM. Although speculative, this procedure might have led to selective averaging of the BOLD response accompanying the start of Nystagmoid-REMs, because they usually appear in the form of REM bursts. Furthermore, even assuming PGO waves as an alternative to visual input from the retina to V1, the neural mechanism that generates vivid and complicated dream scenes is not yet clear. And finally what is the significance of the large difference in the time intervals between REMs and BOLD responses in the various neural structures? These questions will be discussed in relationship to the activation accompanying REMs.

\section{Activation accompanying REM}

Following the activation of the PGO areas, the activation of PHG, ACC, amygdala, and putamen accompanied REMs. 
These spatial activation patterns are fairly consistent with existing PET study results showing increases in regional blood flow or the glucose metabolism of the pontine tegmentum, ventroposterior thalamus, limbic/paralimbic areas, and visual cortex during REM sleep (Maquet et al. 1996; Nofzinger et al. 1997; Peigneux et al. 2001; Braun et al. 1998). A recent fMRI study also shows that a thalamocortical network including limbic and parahippocampal areas is specifically active during phasic REM periods (Wehrle et al. 2007). Our present event-related fMRI study supports and extends this notion. That is, the activated areas do not necessarily remain active throughout REM sleep, but their activation is temporally related to REMs. Among these activated areas, the putamen and ACC, including the SEF, were also activated by self-paced saccades in total darkness in the present and previous fMRI/PET studies (Petit et al. 1993; Gagnon et al. 2002). Therefore, these activations most likely relate to motor control processing that is common to saccades and REMs.

More importantly, REMs temporarily activated the amygdala and PHG, brain regions that self-paced saccades in total darkness did not activate. Recent human and animal studies on REM sleep, visual imagery and memory processing offer us the following two possibilities. First, since the amygdala (Pare et al. 2002; McGaugh et al. 2002) and PHG (Gabrieli et al. 1997; Suzuki et al. 1993) are profoundly associated with memory processing during wakefulness and were not activated in conjunction with self-paced saccades in total darkness, these areas' activation may be associated with a function of REM sleep. It may, according to several human (Maquet et al. 1996, 2000; Peigneux et al. 2003; Stickgold et al. 2001; Wagner et al. 2001) and animal (Cahill and McGaugh 1998; Louie and Wilson 2001) studies, facilitate a consolidation process of emotional and/or implicit memory acquired during the preceding period of wakefulness. In this line of thought, it is interesting to note that we found no significant activation in the hippocampus, though both the amygdala and parahippocampal gyrus were activated. Although the reason for the inactivity of the hippocampus is not clear, this result is consistent with many recent PET (Maquet et al. 1996; Braun et al. 1998; Peigneux et al. 2001) and fMRI (Wehrle et al. 2007) studies. A second possibility is that these activations relate to the generation and maintenance of dream imagery. This possibility is supported by a finding that single neurons in the human medial temporal lobe (MTL: the hippocampus, amygdala, entorhinal cortex and PHG) show selective changes in firing rate while subjects viewed figures and when they were visually recalling the images (Kreiman et al. 2000). This study suggests that there is a common neural substrate for the processing of visual perception and visual imagery, not only in the visual cortex (Kosslyn et al. 1999; Klein et al. 2000), but also in MTL. The activated areas temporally related to
REMs in the present study include these two neural substrates. In fact, electrical stimulation of the amygdala, hippocampus and cingulate gyrus in humans induces not elementary sensations, but sensations of dreaming and hallucination (Halgren et al. 1978), and there is strong correlation between the number of REMs and visual imagery in dreaming (Hong et al. 1997). These findings are also consistent with the notion that the activation of V1 and the subsequent activation of the amygdala and PHG reflect neural activities for generating and maintaining vivid and complex dream scenes. In either case, it is suggested that REMs are not merely an epiphenomenon of PGO waves, but REMs and/or their generating mechanism may be essentially involved in triggering activation of the amygdala and PHG and therefore the function of REM sleep.

An intriguing but puzzling issue is the varying time intervals between BOLD responses and REMs in different neural structures. It is well known that there are anatomical and physiological correlations between the amygdala and the pons, where the PGO wave generator is located (Calvo and Fernandez-Guardiola 1984; Datta et al. 1998) and that the time interval between PGO waves and REMs is on the order of tens of milliseconds in neurophysiological studies using animals (Datta and Hobson 1994). In contrast, the BOLD response of the PGO areas started several seconds before the occurrence of REMs in the present study. First of all, it should be discussed whether the time intervals between BOLD responses in the different neural structures and REMs actually reflect the temporal difference of corresponding neural activities. Although it is still controversial whether BOLD response timing is sufficient for determining the timing of neural activity in different brain regions (Friston et al. 1998; Miezin et al. 2000), such a large difference in timing and the following observations lead us to suggest that the BOLD response in PGO areas resulted from neural activity that actually preceded REM onset. First, it is well known that the rising edge of a BOLD response is the most precise indicator of BOLD onset (Menon and Kim 1999), and the rising edges of the PGO area responses evidently preceded REM onset (Fig. 4a). Secondly, the signal increase in other areas that accompanied REM onset rules out the possibility that the grand-averaged data were contaminated with BOLD responses caused by imperceptibly small REMs that preceded the REMs used for the averaging. Finally, unlike the usual case in fMRI experiments where stimuli are experimentally presented, REMs occur spontaneously during sleep. In the case of such a spontaneous event, it is reasonable to postulate that the neural activity involved in generating the event and the corresponding BOLD response started before the event. In fact, Hawco et al. (2007) also reported that a BOLD response without discernible scalp EEG changes starts several seconds before spontaneous epileptic spikes. Therefore, it is plausible that 
the onset time of the BOLD response actually precedes REM onset. However, considering the hemodynamic delay between neural activity and the onset of the corresponding BOLD response (typically 1-2 s) and the recent MEG/EEG studies that reported neural activities immediately before REMs in the several brain areas including the anterior cingulate cortex, parahippocampal gyrus, amygdala and so on (Ioannides et al. 2004; Abe et al. 2008), we cannot fully eliminate the possibility that neural activities not only in the PGO areas but also in other activated areas start just before REM onset. Even in that case, there were significantly large differences in the onset time between the PGO areas and other cortical/subcortical areas.

Then what could be the significance of such large variations in the time interval between BOLD responses and REMs across neural structures? Since the differences seen here are much larger than those seen in animal studies, the preceding activation of PGO-areas may not reflect the direct neuron-to-neuron electrophysiological process revealed by animal studies, but rather a shift in the brain from a relatively tonic state to another state. REM sleep is known to contain tonic and phasic periods. The phasic REM period is characterized by REM bursts and other phasic events, whereas almost no REMs occur during the tonic REM period. Since we used only the first REM of REM bursts and isolated REMs for the time course analysis, the preceding activation of the PGO areas (sometimes by several seconds) may reflect a switch in the brain from tonic REM to phasic REM. Although there is no direct evidence to support this idea, the finding that most neurons in the caudolateral peribrachial area begin to increase their firing rates several seconds before the first PGO wave in the PGO-related state (Datta and Hobson 1994) supports this notion. An alternative and/or supplementary explanation for the large variations in the time interval between BOLD responses and REMs, especially for the preceding activation of the thalamus and V1, is that different brain regions have different hemodynamic response latencies (Buckner et al. 1996; Menon and Kim 1999).

Finally, it should be noted that most neurophysiological studies on PGO waves and REM sleep have been conducted in cats, and PGO waves or PGO-like wave activity has been reported in a number of other mammals as well, including rats, nonhuman primates and humans. However, the spatial distribution of PGO waves in the brain is different in different species. In particular, the inactivity of the hippocampus in humans shown in the present and previous PET studies is a contrast to results of animal studies that reported neuronal activity in the hippocampus during REM sleep (Poe et al. 2000; Louie and Wilson 2001). Furthermore, the temporal relation of PGO waves to REMs across species is also not clear yet. That is, whereas REMs are highly correlated with PGO-waves in the cat (Nelson et al.
1983; Datta and Hobson 1994), PGO-waves in humans are incompletely associated with REMs (Lim et al. 2007). The present study emphasizes that further research will be needed to more fully understand the functional neuroanatomy of PGO waves and REMs across species.

\section{Conclusions}

In conclusion, using simultaneous polysomnographic recording with fMRI and event-related fMRI analysis timelocked to REMs, we succeeded in identifying the human cortical-subcortical network, which is not necessarily activated throughout REM sleep, but is phasically activated with the occurrence of REMs. The present findings may not only help our understanding of sleep physiology in humans, but they may also provide clues for understanding the relationship between REMs and dreaming.

Acknowledgments We thank Dr. Hiroshige Takeichi and Dr. Robert Turner for their useful suggestions. This work was partly supported by CREST of Japan Science and Technology (JST).

Open Access This article is distributed under the terms of the Creative Commons Attribution Noncommercial License which permits any noncommercial use, distribution, and reproduction in any medium, provided the original author(s) and source are credited.

\section{References}

Abe T, Ogawa T, Nittono H, Hori T (2008) Neural generators of brain potentials before rapid eye movements during human REM sleep: a study using sLORETA. Clin Neurophysiol 119:2044-2053

Baillet S, Mosher JC, Leahy RM (2001) Electromagnetic brain mapping. IEEE Signal Process Mag 18:14-30

Birn RM, Cox RW, Bandettini PA (2002) Detection versus estimation in event-related fMRI: choosing the optimal stimulus timing. NeuroImage 15:252-264

Bodis-Wollner I, Bucher SF, Seelos KC, Paulus W, Reiser M, Oertel WH (1997) Functional MRI mapping of occipital and frontal cortical activity during voluntary and imagined saccades. Neurology 49:416-420

Brandt SA, Stark LW (1997) Spontaneous eye movements during visual imagery reflect the content of the visual scene. J Cogn Neurosci 9:27-38

Braun AR, Balkin TJ, Wesensten NJ, Gwadry F, Carson RE, Varga M, Baldwin P, Belenky G, Herscovitch P (1998) Dissociated pattern of activity in visual cortices and their projections during human rapid eye movement sleep. Science 279:91-95

Bristow D, Haynes JD, Sylvester R, Frith CD, Rees G (2005) Blinking suppresses the neural response to unchanging retinal stimulation. Curr Biol 15:1296-1300

Buckner RL, Bandettini PA, O’Craven KM, Savoy RL, Petersen SE, Raichle ME, Rosen BR (1996) Detection of cortical activation during averaged single trials of a cognitive task using functional magnetic resonance imaging. Proc Natl Acad Sci USA 93:1487814883

Cahill L, McGaugh L (1998) Mechanisms of emotional arousal and lasting declarative memory. Trends Neurosci 21:294-299 
Callaway CW, Lydic R, Baghdoyan HA, Hobson JA (1987) Pontogeniculoccipital waves: spontaneous visual system activity during rapid eye movement sleep. Cell Mol Neurobiol 7:105-149

Calvo JM, Fernandez-Guardiola A (1984) Phasic activity of the basolateral amygdala, cingulate gyrus, and hippocampus during REM sleep in the cat. Sleep 7:202-210

Datta S, Hobson AJ (1994) Neuronal activity in the caudolateral peribrachial pons: relationship to PGO waves and rapid eye movements. J Neurophysiol 71:95-109

Datta S (2000) Avoidance task training potentiates phasic pontinewave density in the rat: a mechanism for sleep-dependent plasticity. J Neurosci 20:8607-8613

Datta S, Siwek DF, Patterson EH, Cipolloni PB (1998) Localization of pontine PGO wave generation sites and their anatomical projections in the rat. Synapse 30:409-423

Dement WC, Kleitman N (1957) The relation of eye movements during sleep to dream activity: an objective method for the study of dreaming. J Exp Psychol 53:339-346

Doricchi F, Guariglia C, Paolucci S, Pizzamiglio L (1993) Disturbances of the rapid eye movements (REMs) of REM-sleep in patients with unilateral attentional neglect: clue for the understanding of the functional meaning of REMs. Clin Neurophysiol 87:105-116

Doricchi F, Guariglia C, Paolucci S, Pizzamiglio L (1996) Asymmetry of rapid eye movements in chronic unilateral neglect does not change with behavioral improvement induced by rehabilitation treatment. Clin Neurophysiol 98:51-58

Doricchi F, Iaria G, Silvetti M, Figliozzi F, Siegler I (2007) The "ways" we look at dreams: evidence from unilateral spatial neglect (with an evolutionary account of dream bizarreness). Exp Brain Res 178:450-461

Friston KJ, Fletcher P, Josephs O, Holmes A, Rugg MD, Turner R (1998) Event-related fMRI: characterizing differential responses. NeuroImage 7:30-40

Friston KJ, Zarahn E, Josephs O, Henson RNA, Dale AM (1999) Stochastic designs in event-related fMRI. NeuroImage 10:607-619

Gabrieli JDE, Brewer JB, Desmond JE, Glover GH (1997) Separate neural bases of two fundamental memory processes in the human medial temporal lobe. Science 276:264-266

Gagnon D, O'Driscoll GA, Petrides M, Pike GB (2002) The effect of spatial and temporal information on saccades and neural activity in oculomotor structures. Brain 125:123-139

Halgren E, Walter RD, Cherlow DG, Crandall PH (1978) Mental phenomena evoked by electrical stimulation of the human hippocampal formation and amygdala. Brain 101:83-117

Hawco CS, Bagshaw AP, Lu Y, Dubeau F, Gotman J (2007) BOLD signal occur prior to epileptic spikes seen on scalp EEG. NeuroImage 35:1450-1458

Hobson JA, McCarley RW (1977) The brain as a dream state generator: an activation-synthesis hypothesis of the dream process. Am J Psychiatry 134:1335-1348

Hobson JA, Pace-Schott EF, Stickgold R (2000) Dreaming and the brain: toward a cognitive neuroscience of conscious states. Behav Brain Sci 23:793-842

Hong CCH, Potkin SC, Antrobus JS, Dow BM, Callaghan CM, Gillin JC (1997) REM sleep eye movement counts correlate with visual imagery in dreaming: a pilot study. Psycholphysiology 34:377381

Ioannides AA, Corsi-Cabrera M, Fenwick PBC, Portilla YR, Laskaris NA, Khurshudyan A, Theofilou D, Shibata T, Uchida S, Nakabayashi T, Kostopoulos GK (2004) MEG tomography of human cortex and brainstem activity in waking and REM-sleep saccades. Cereb Cortex 14:56-72

Kato M, Miyauchi S (2003) Functional MRI of brain activation evoked by intentional eye blinking. NeuroImage 18:749-759
Klein I, Paradis AL, Poline JB, Kosslyn SM, Le Bihan D (2000) Transient activity in the human calcarine cortex during visual-mental imagery: an event-related fMRI study. J Cogn Neurosci 12(suppl) 2:15-23

Kosslyn SM, Pascual-Leone A, Felician O, Camposano S, Keenan JP, Thompson WL, Ganis G, Sukel KE, Alpert NM (1999) The role of area 17 in visual imagery: convergent evidence from PET and rTMS. Science 284:167-170

Kreiman G, Koch C, Fried I (2000) Imagery neurons in the human brain. Nature 408:357-361

Laeng B, Teodorescu D-S (2002) Eye scanpaths during visual imagery reenact those of perception of the same visual scene. Cogn Sci 26:207-231

Lim AS, Lozano AM, Moro E, Hamani C, Hutchison WD, Dostrovsky JO, Lang AE, Wennberg RA, Murray BJ (2007) Characterization of REM-sleep associated ponto-geniculo-occipital waves in the human pons. Sleep 30:823-827

Louie K, Wilson MA (2001) Temporally structured replay of awake hippocampal ensemble activity during rapid eye movement sleep. Neuron 29:145-156

Maquet P, Peters JM, Aerts J, Delfiore G, Degueldre C, Luxen A, Franck G (1996) Functional neuroanatomy of human rapid-eyemovement sleep and dreaming. Nature 383:163-166

Maquet P, Laureys S, Peigneux P, Fuchs S, Petiau C, Phillips C, Aerts J, Gelfiore G, Degueldre C, Luxen A, Franck G, van der Linden M, Smith C, Cleeremans A (2000) Experience-dependent changes in cerebral activation during human REM-sleep. Nat Neurosci 3:831-836

Marks A, Shaffery JP, Oksenberg A, Speciale SG, Roffwarg HP (1995) A functional role for REM-sleep in brain maturation. Behav Brain Res 69:1-11

McGaugh JL, McIntyre CK, Power AE (2002) Amygdala modulation of memory consolidation: interaction with other brain systems. Neurobiol Learn Mem 78:539-552

Menon RS, Kim S-G (1999) Spatial and temporal limits in cognitive neuroimaging with fMRI. Trends Cogn Sci 3:207-216

Miezin FM, Maccotta L, Petersen Ollinger JM, SE Buckner RL (2000) Characterizing the hemodynamic response: effects of presentation rate, sampling procedure, and the possibility of ordering brain activity based on relative timing. NeuroImage 11:735-759

Miyauchi S, Takino R, Azakami M (1990) Evoked potentials during REM-sleep reflect dreaming. Clin Neurophysiol 76:19-28

Nofzinger EA, Mintun MA, Wiseman M, Kupfer DJ, Moore RY (1997) Forebrain activation in REM sleep: an FDG PET study. Brain Res 770:192-201

Nelson JP, McCarley RW, Hobson JA (1983) REM sleep burst neurons, PGO waves, and eye movement information. J Neurophysiol 50:784-797

Ogawa K, Nittono H, Hori T (2005) Brain potentials before and after rapid eye movements: an electrophysiological approach to dreaming in REM-sleep. Sleep 28:1077-1082

Pare D, Collins DR, Pelletier JG (2002) Amygdala oscillations and the consolidation of emotional memories. Trends Cogn Sci 6:306314

Peigneux P, Laureys S, Fuchs S, Delbeuck X, Degueldre C, Aerts J, Delfiore G, Luxen A, Maquet P (2001) Generation of rapid eye movements during paradoxical sleep in humans. NeuroImage 14:749-758

Peigneux P, Laureys S, Fuchs S, Destrebecqz A, Collette F, Felbeuck X, Phillips C, Aerts J, Delfiore G, Degueldre C, Luxen A, Cleeremans A, Maquet P (2003) Learned material content and acquisition level modulate cerebral reactivation during posttraining rapid-eye-movements sleep. NeuroImage 20:125-134

Petit L, Orssaud C, Tzourio N, Salamon G, Mazoyer B, Berthoz A (1993) PET study of voluntary saccadic eye movements in hu- 
mans: basal ganglia-thalamocortical system and cingulate cortex involvement. J Neurophysiol 69:1009-1017

Poe GR, Nitz DA, McNaughton BL, Barnes CA (2000) Experiencedependent phase-reversal of hippocampal neuron firing during REM sleep. Brain Res 855:176-180

Rechtschaffen A, Kales AA (1968) A manual of standardized terminology, techniques and scoring system for sleep stages of human subjects. Government Printing Office, Washington DC

Redcay E, Kennedy DP, Courchesne E (2007) fMRI during natural sleep as a method to study brain function during early childhood. NeuroImage 38:696-707

Roffwarg HP, Dement WC, Muzio JN, Fisher C (1962) Dream imagery: relationship to rapid eye movements of sleep. Arch Gen Psychiatry 7:235-258

Salzarulo P, Lairy GC, Bancaud J, Munari C (1975) Direct depth recording of the striate cortex during REM-sleep in man: Are there PGO potentials? Clin Neurophysiol 38:199-202

Stickgold R, Hobson JA, Fosse R, Fosse M (2001) Sleep, learning, and dreams: off-line memory reprocessing. Science 294:1052-1057
Suzuki WA, Zola-Morgan S, Squire LR, Amaral DG (1993) Lesions of the perirhinal and parahippocampal cortices in the monkey produce long-lasting memory impairment in the visual and tactual modalities. J Neurosci 13:2430-2451

Sylvester R, Rees G (2006) Extraretinal saccadic signals in human LGN and early retinotopic cortex. NeuroImage 30:214-219

Wagner U, Gais S, Born J (2001) Emotional memory formation is enhanced across sleep intervals with high amounts of rapid eye movement sleep. Learn Mem 8:112-119

Wehrle R, Czisch M, Kaufmann C, Wetter TC, Holaboer F, Auer DP, Pollmächer T (2005) Rapid eye movement-related brain activation in human sleep: a functional magnetic resonance imaging study. NeuroReport 16:853-857

Wehrle R, Kaufmann C, Wetter TC, Holsboer F, Auer DP, Pollmacher T, Czisch M (2007) Functional microstates within human REM sleep: first evidence from fMRI of a thalamocortical network specific for phasic REM periods. Eur J NeuroSci 25:863-871 